\title{
On Random Bernoulli Convolutions
}

\author{
Tomas Persson
}

Preprints in Mathematical Sciences

2008:7

LUND INSTITUTE OF TECHNOLOGY Lund University

Centre for Mathematical Sciences

Mathematics 



\title{
On random Bernoulli convolutions
}

\author{
Tomas Persson
}

May 25, 2008

With one figure

\begin{abstract}
We study the distribution of the random series $\sum_{k=0}^{\infty} \pm \lambda_{k}^{k}$ where $\lambda_{k}$ are independently and uniformly distributed in $(\lambda-\varepsilon, \lambda+\varepsilon)$. It is proved that the distribution of the series has density in $L_{2}$ and that the $L_{2}$ norm of the density does not grow faster than $1 / \sqrt{\varepsilon}$.
\end{abstract}

\section{Introduction and Statements of Results}

Let $\lambda \in\left(\frac{1}{2}, 1\right)$. The distribution of the random variable $Y_{\lambda}$, defined by

$$
Y_{\lambda}=(1-\lambda) \sum_{k=0}^{\infty} \vartheta_{k} \lambda^{k}
$$

where the random variables $\vartheta_{k}$ are independent and identically distributed according to $P\left(\vartheta_{k}=+1\right)=P\left(\vartheta_{k}=-1\right)=1 / 2$, is denoted by $\nu_{\lambda}$. We have chosen the constant $(1-\lambda)$ in front of the sum in order to have $\nu_{\lambda}$ with the support $[-1,1]$ for all $\lambda$. This distribution was studied by Erdös in [I] and [2]. It is known that $\nu_{\lambda}$ is absolutely continuous with respect to Lebesgue measure for almost all $\lambda \in\left(\frac{1}{2}, 1\right)$, see [7] and [4], and that if $\lambda^{-1}$ is a Pisot number, then $\nu_{\lambda}$ is singular with respect to Lebesgue measure, [r]. It is also known that for $\lambda=1 / \sqrt[k]{(2),} k \in \mathbb{N}$, and for some algebraic integers, $\nu_{\lambda}$ is absolutely continuous with respect to Lebesgue measure, [3], [9]. There is a nice survey of the subject in [5].

For $\varepsilon>0$, we study the distribution of the random variable

$$
Y_{\lambda, \varepsilon}=(1-\lambda-\varepsilon) \sum_{k=0}^{\infty} \vartheta_{k} \lambda_{k}^{k},
$$

where the random variables $\lambda_{k}$ are uniformly and independently distributed in $(\lambda-$ $\varepsilon, \lambda+\varepsilon)$, and the random variables $\vartheta_{k}$ are independent, independent of $\lambda_{k}$ and distributed as above. We let $\nu_{\lambda, \varepsilon}$ denote the distribution of the random variable $Y_{\lambda, \varepsilon}$. The coefficient $1-\lambda-\varepsilon$ in the definition of $Y_{\lambda, \varepsilon}$ is chosen in order that the measure $\nu_{\lambda, \varepsilon}$ has support in $[-1,1]$. 
It is not hard to see that the distribution $\nu_{\lambda, \varepsilon}$ approximates $\nu_{\lambda}$. More precisely, one easily proves the following theorem.

Theorem I. The measure $\nu_{\lambda, \varepsilon}$ converges weakly to the measure $\nu_{\lambda}$ as $\varepsilon \rightarrow 0$.

Since $\nu_{\lambda}$ is absolutely continuous with respect to Lebesgue measure for almost all $\lambda$ between $\frac{1}{2}$ and 1 , but not for all $\lambda$, it is natural to ask how regular $\nu_{\lambda, \varepsilon}$ is. We will prove that $\nu_{\lambda, \varepsilon}$ has density in $L_{2}$ and that the $L_{2}$ norm of the density can not grow faster that $1 / \sqrt{\varepsilon}$. Let $\mathcal{L}$ denote the Lebesgue measure on $\mathbb{R}$. The following theorems will be proved.

Theorem 2. For any $\delta>0$ there exists a constant $c$ such that for any $\varepsilon>0$ there is a set $E \subset\left(\frac{1}{2}+\varepsilon, 0.6491-\varepsilon\right)$ with $\mathcal{L}\left(\left(\frac{1}{2}+\varepsilon, 0.6491-\varepsilon\right) \backslash E\right)<\delta$, such that the measure $\nu_{\lambda, \varepsilon}$ satisfies $\left\|\nu_{\lambda, \varepsilon}\right\|_{2}<c$ for any $\lambda \in E$.

Theorem 3. If $\lambda \in\left(\frac{1}{2}, 1\right)$, then the density of $\nu_{\lambda, \varepsilon}$ is in $L_{2}$ and $\left\|\nu_{\lambda, \varepsilon}\right\|_{2}<c / \sqrt{\varepsilon}$, for all $0<\varepsilon<\max \left\{\lambda-\frac{1}{2}, 1-\lambda\right\}$, where $c=\frac{4 \lambda \sqrt{\lambda}}{\sqrt{\lambda^{2}-\frac{1}{4}-(2 \lambda+1) \varepsilon}}$.

Theorem 2 is proved in Section 2. The proof is only a minor modification of the proof in Peres' and Solomyak's article [4]. The proof of Theorem 3 is in Section 3. It is based on the ideas of transversal intersection of unstable curves in Tsujii's article [8].

\section{Proof of Theorem 2}

Let $Q=[-1,1)^{3}$ and $m \in \mathbb{N}$. We partition the cube $Q$ into the parallelepipeds $\left\{Q_{0, k}, Q_{1, k}\right\}_{k=0}^{2^{m}-1}$, where

$$
\begin{aligned}
& Q_{0, k}=\left\{(x, y, z) \in Q: y<0,-1+k 2^{-m+1} \leq z<-1+(k+1) 2^{-m+1}\right\}, \\
& Q_{1, k}=\left\{(x, y, z) \in Q: y \geq 0,-1+k 2^{-m+1} \leq z<-1+(k+1) 2^{-m+1}\right\} .
\end{aligned}
$$

Define $f_{\lambda, \varepsilon, m}: Q \rightarrow Q$ by

$$
f_{\lambda, \varepsilon, m}:(x, y, z) \mapsto\left(\lambda_{0}(z) x+a(y, z), 2 y+b(y), 2^{m} z+c(z)\right),
$$

where $\lambda_{0}(z)$ and $a(y, z), b(y), c(z)$ are defined by

$$
\begin{aligned}
& \lambda_{0}(z)=\lambda+2^{m+1} \varepsilon\left(z-\left(-1+\left(k+\frac{1}{2}\right) 2^{-m+1}\right)\right), \quad(x, y, z) \in Q_{0, k} \cup Q_{1, k} \\
& a(y, z)=\left\{\begin{aligned}
-1+\lambda_{0}(z) & \text { if }(x, y, z) \in Q_{0, k} \\
1-\lambda_{0}(z) & \text { if }(x, y, z) \in Q_{1, k}
\end{aligned}\right. \\
& b(y)=\left\{\begin{array}{rl}
1 & \text { if }(x, y, z) \in Q_{0, k} \\
-1 & \text { if }(x, y, z) \in Q_{1, k}
\end{array},\right. \\
& c(z)=2^{m}-2 k-1, \quad(x, y, x) \in Q_{0, k} \cup Q_{1, k} .
\end{aligned}
$$

There is a picture of the map $f_{\lambda, \varepsilon, 2}$ in Figure $\mathrm{I}$.

For later use we define the sets

$$
Q_{0}=\bigcup_{k=0}^{2^{m}-1} Q_{0, k}, \quad Q_{1}=\bigcup_{k=0}^{2^{m}-1} Q_{1, k} .
$$



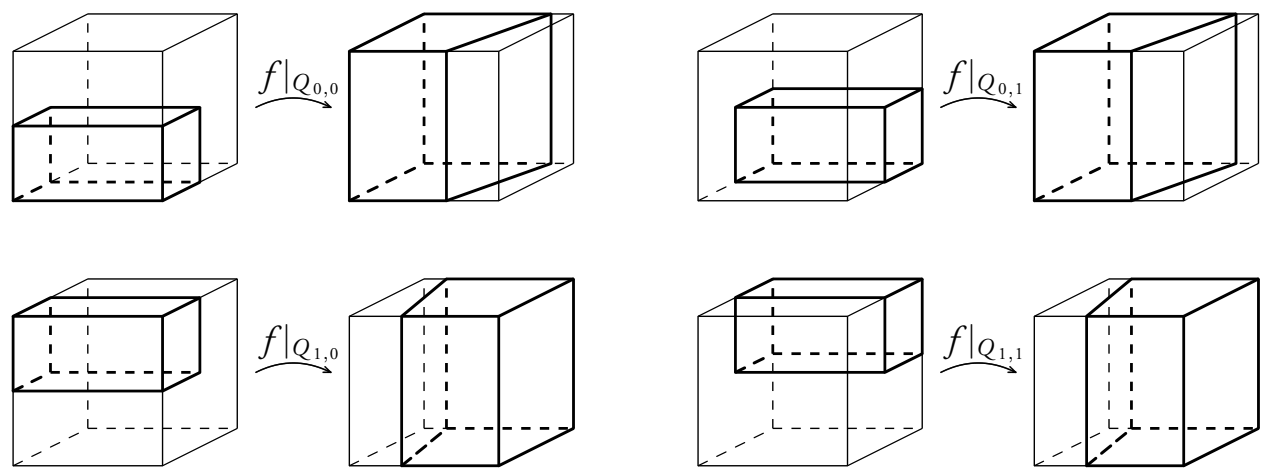

Figure r: The action of the map $f_{\lambda, \varepsilon, 2}$ restricted to the sets $Q_{0,0}, Q_{0,1}, Q_{1,0}$ and $Q_{1,1}$, for $\lambda=3 / 4$ and $\varepsilon=1 / 10$.

Let $\nu$ be the normalised Lebesgue measure on $Q$. The measures

$$
\mu_{\lambda, \varepsilon, m, n}=\frac{1}{n} \sum_{k=0}^{n-1} \nu \circ f_{\lambda, \varepsilon, m}^{-k},
$$

converges weakly to an SRB-measure $\mu_{\lambda, \varepsilon, m}$ as $n \rightarrow \infty$. The measure $\mu_{\lambda, \varepsilon, m}$ is clearly ergodic, since if $p=\left(x_{0}, y_{0}, z_{0}\right) \in Q$, then the stable manifold of $p$ is

$$
\left\{(x, y, z) \in Q: y=y_{0}, z=z_{0}\right\},
$$

and the unstable manifold of $p$ is

$$
\left\{(x, y, z) \in Q: x=x_{0}\right\} .
$$

Moreover, the projection of $\mu_{\lambda, \varepsilon, m}$ onto the first coordinate is a measure $\nu_{\lambda, \varepsilon, m}$. More precisely, if $E \subset[-1,1)$ is a measurable set, then $\nu_{\lambda, \varepsilon, m}(E)=\mu_{\lambda, \varepsilon, m}(E \times$ $[-1,1) \times[-1,1))$, defines a measure on $[-1,1)$.

The measure $\nu_{\lambda, \varepsilon, m}$ is the distribution of a powerseries $(1-\lambda-\varepsilon) \sum_{k=0}^{\infty} \vartheta_{k} \lambda_{k}^{k}$ where $\lambda_{k}$ are uniformly distributed in $(\lambda-\varepsilon, \lambda+\varepsilon)$, but not independent. However, the measure $\nu_{\lambda, \varepsilon, m}$ converges weakly to $\nu_{\lambda, \varepsilon}$ as $m \rightarrow \infty$.

Let $A$ be a set of $2^{m+1}$ elements. We denote the elements in $A$ in such a way that $A=\left\{(0,0),(0,1), \ldots,\left(0,2^{m}-1\right),(1,0),(1,1), \ldots,\left(1,2^{m}-1\right)\right\}$. Put $A_{0}=$ $\left\{(0,0),(0,1), \ldots,\left(0,2^{m}-1\right)\right\}$ and $A_{1}=\left\{(1,0),(1,1), \ldots,\left(1,2^{m}-1\right)\right\}$.

Let $\Sigma_{0}=A^{\mathbb{N} \cup\{0\}}$. If $p$ is a point in $Q$ then there is a unique sequence $\rho_{0}(p)=$ $\left\{\rho_{0}(p)_{k}\right\}_{k=0}^{\infty} \in \Sigma_{0}$ such that

$$
f_{\lambda, \varepsilon, m}(p) \in Q_{\rho_{0}(p)_{k}}, \quad k=0,1, \ldots
$$

The map $\rho_{0}: Q \rightarrow \Sigma_{0}$ is not injective.

We can transfere the measures $\mu_{\lambda, \varepsilon, m}$ and $\nu_{\lambda, \varepsilon, m}$ to measures $\mu_{\Sigma_{0}}$ and $\nu_{\Sigma_{0}}$ on $\Sigma_{0}$ by $\mu_{\Sigma_{0}}=\mu_{\lambda, \varepsilon, m} \circ \rho_{0}^{-1}$ and $\nu_{\Sigma_{0}}=\nu_{\lambda, \varepsilon, m} \circ \rho_{0}^{-1}$. 
We let $\Sigma$ denote the natural extension of $\Sigma_{0}$. That is, $\Sigma$ is the set of all two sided infinite sequences such that any one sided infinite subsequence of a sequence in $\Sigma$ is a sequence in $\Sigma_{0}$. The measure $\mu_{\Sigma_{0}}$ defines an ergodic measure $\mu_{\Sigma}$ on $\Sigma$ in a natural way. If $\eta: \Sigma \rightarrow \Sigma_{0}$ is defined by $\eta\left(\left\{i_{k}\right\}_{k \in \mathbb{Z}}\right)=\left\{i_{k}\right\}_{k \in \mathbb{N} \cup\{0\}}$, then $\mu_{\Sigma_{0}}(E)=\mu_{\Sigma}\left(\eta^{-1}(E)\right)$. Hence, if a function $\varphi: \Sigma_{0} \rightarrow \mathbb{R}$ is $\mu_{\Sigma_{0}}$-measurable, then the function $\varphi \circ \eta: \Sigma \rightarrow \mathbb{R}$ is $\mu_{\Sigma}$-measurable. The map $\rho_{0}: Q \rightarrow \Sigma_{0}$ can be extended to a map $\rho: Q \rightarrow \Sigma$ such that $\rho(\sigma(a))=f_{\lambda, \varepsilon, m}(\rho(a))$ holds for any sequence $a \in \Sigma$.

Let $B(x, r)=[x-r, x+r]$ be the interval of radius $r$ around $x$. The limit

$$
D(x, \lambda, \varepsilon)=\liminf _{r \rightarrow 0} \frac{\nu_{\lambda, \varepsilon, m}(B(x, r))}{2 r}
$$

is the density of the measure $\nu_{\lambda, \varepsilon}$ if it exists. If

$$
\int_{-1}^{1} D(x, \lambda, \varepsilon) \mathrm{d} \nu_{\lambda, \varepsilon, m}(x)<\infty
$$

then the density $D(x, \lambda, \varepsilon)$ is in $L_{2}$. We prove that

$$
\int_{\frac{1}{2}+\varepsilon+\delta}^{0.6491-\varepsilon-\delta} \int_{-1}^{1} D(x, \lambda, \varepsilon) \mathrm{d} \nu_{\lambda, \varepsilon, m}(x) \mathrm{d} \lambda<C,
$$

for any $\varepsilon>\delta>0$ and some constant $C$ which is independent of $m$. This implies that $D(x, \lambda, \varepsilon)$ is in $L_{2}$ for almost all $\lambda \in\left(\frac{1}{2}+\varepsilon, 1-\varepsilon\right)$, which implies the statement of Theorem 2.

By Fatou's lemma, we get the following inequality.

$$
\begin{aligned}
I & =\int_{\frac{1}{2}+\varepsilon+\delta}^{0.6491-\varepsilon-\delta} \int_{-1}^{1} D(x, \lambda, \varepsilon) \mathrm{d} \nu_{\lambda, \varepsilon, m}(x) \mathrm{d} \lambda \\
& =\int_{\frac{1}{2}+\varepsilon+\delta}^{0.6491-\varepsilon-\delta} \int_{-1}^{1} \liminf _{r \rightarrow 0} \frac{\nu_{\lambda, \varepsilon, m}(B(x, r))}{2 r} \mathrm{~d} \nu_{\lambda, \varepsilon}(x) \mathrm{d} \lambda \\
& \leq \liminf _{r \rightarrow 0} \frac{1}{2 r} \int_{\frac{1}{2}+\varepsilon+\delta}^{0.6491-\varepsilon-\delta} \int_{-1}^{1} \nu_{\lambda, \varepsilon, m}(B(x, r)) \mathrm{d} \nu_{\lambda, \varepsilon, m}(x) \mathrm{d} \lambda \\
& =\liminf _{r \rightarrow 0} \frac{1}{2 r} \int_{\frac{1}{2}+\varepsilon+\delta}^{0.6491-\varepsilon-\delta} \int_{-1}^{1} \int_{-1}^{1} I_{\{z:|x-z|<r\}}(y) \mathrm{d} \nu_{\lambda, \varepsilon, m}(y) \mathrm{d} \nu_{\lambda, \varepsilon, m}(x) \mathrm{d} \lambda
\end{aligned}
$$

where $I$ denotes the indicator function.

Since $\nu_{\lambda, \varepsilon, m}$ is the projection of $\mu_{\lambda, \varepsilon, m}$ to the first coordinate we can rewrite the last line as integrals over the measure $\mu_{\lambda, \varepsilon, m}$ to get the estimate

$$
I \leq \liminf _{r \rightarrow 0} \frac{1}{2 r} \int_{\frac{1}{2}+\varepsilon+\delta}^{0.6491-\varepsilon-\delta} \int_{Q} \int_{Q} I_{\left\{z:\left|x_{1}-z_{1}\right|<r\right\}}(y) \mathrm{d} \mu_{\lambda, \varepsilon, m}(y) \mathrm{d} \mu_{\lambda, \varepsilon, m}(x) \mathrm{d} \lambda,
$$

where $x_{1}$ and $z_{1}$ denotes the first coordinate of the points $x, z \in Q$. 
We can transfere the two innermost integrals to integrals over $\Sigma$ in order to get the estimate

$$
\begin{aligned}
I & \leq \liminf _{r \rightarrow 0} \frac{1}{2 r} \int_{\frac{1}{2}+\varepsilon+\delta}^{0.6491-\varepsilon-\delta} \int_{\Sigma} \int_{\Sigma} I_{\left.\left\{c: \mid \rho(a)_{1}-\rho(c)\right)_{1} \mid<r\right\}}(b) \mathrm{d} \mu_{\Sigma}(b) \mathrm{d} \mu_{\Sigma}(\boldsymbol{a}) \mathrm{d} \lambda \\
& =\liminf _{r \rightarrow 0} \frac{1}{2 r} \int_{\frac{1}{2}+\varepsilon+\delta}^{0.6491-\varepsilon-\delta} \int_{\Sigma \times \Sigma} I_{\left\{(a, b):\left|\rho(a)_{1}-\rho(b)_{1}\right|<r\right\}} \mathrm{d}\left(\mu_{\Sigma} \times \mu_{\Sigma}\right) \mathrm{d} \lambda
\end{aligned}
$$

The product space $\Sigma \times \Sigma$ can be written as

$$
\Sigma \times \Sigma=\bigcup_{n=0}^{\infty} \bigcup_{a_{-n} \cdots a_{0}} \bigcup_{\begin{array}{c}
b_{-n} \cdots b_{0} \\
a_{k}, b_{k} \in A_{0} \text { or } a_{k}, b_{k} \in A_{1}, k>-n \\
\left\{a_{-n}, b_{-n}\right\} \not \subset A_{0} \text { and }\left\{a_{-n}, b_{-n}\right\} \not A_{1}
\end{array}}\left[a_{-n} \cdots a_{0}\right] \times\left[b_{-n} \cdots b_{0}\right] .
$$

Hence

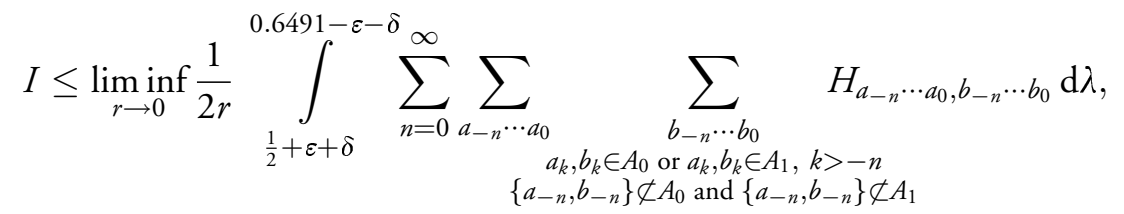

where

$$
H_{a_{-n} \cdots a_{0}, b_{-n} \cdots b_{0}}=\int_{\left[a_{-n} \cdots a_{0}\right] \times\left[b_{-n} \cdots b_{0}\right]} I_{\left\{(a, b):\left|\rho(a)_{1}-\rho(b)_{1}\right|<r\right\}} \mathrm{d}\left(\mu_{\Sigma} \times \mu_{\Sigma}\right) .
$$

We can change order of integration to obtain

$$
\begin{aligned}
& I \leq \liminf _{r \rightarrow 0} \frac{1}{2 r} \sum_{n=0}^{\infty} \sum_{a_{-n} \cdots a_{0}} \quad \sum_{b_{-n} \cdots b_{0}} \quad J_{a_{-n} \cdots a_{0}, b_{-n} \cdots b_{0}}, \\
& \begin{array}{c}
a_{k}, b_{k} \in A_{0} \text { or } a_{k}, b_{k} \in A_{1}, k>-n \\
\left\{a_{-n}, b_{-n}\right\} \not \subset A_{0} \text { and }\left\{a_{-n}, b_{-n}\right\} \not \subset A_{1}
\end{array}
\end{aligned}
$$

where

$$
\begin{aligned}
& J_{a_{-n} \cdots a_{0}, b_{-n} \cdots b_{0}} \\
& =\int_{\left[a_{-n} \cdots a_{0}\right] \times\left[b_{-n} \cdots b_{0}\right]} \int_{\frac{1}{2}+\varepsilon+\delta}^{0.6491-\varepsilon-\delta} I_{\left\{(\boldsymbol{a}, b):\left|\rho(a)_{1}-\rho(b)_{1}\right|<r\right\}} \mathrm{d} \lambda \mathrm{d}\left(\mu_{\Sigma} \times \mu_{\Sigma}\right)(\boldsymbol{a}, \boldsymbol{b})
\end{aligned}
$$

If $\boldsymbol{a}$ and $\boldsymbol{b}$ are two sequences in $\Sigma$ with $\rho(\boldsymbol{a})=\left(x_{\boldsymbol{a}}, y_{\boldsymbol{a}}, z_{\boldsymbol{a}}\right)$ and $\rho(\boldsymbol{b})=\left(x_{\boldsymbol{b}}, y_{\boldsymbol{b}}, z_{\boldsymbol{b}}\right)$, then

$$
x_{a}=\sum_{k=0}^{\infty} \lambda_{0}^{k}\left(\lambda_{0}-1\right)(-1)^{a_{-k-1}}
$$


If $\boldsymbol{a}$ and $\boldsymbol{b}$ are such that for $-n<k<0$ it holds that either $a_{k}, b_{k} \in A_{0}$ or $a_{k}, b_{k} \in A_{1}$, and $a_{-n} \in A_{0}$ and $b_{-n} \in A_{1}$, then

$$
x_{a}-x_{b}=\sum_{k=n}^{\infty} \lambda_{0}^{k}\left(\lambda_{0}-1\right)\left((-1)^{a_{-k}}-(-1)^{b_{-k}}\right) .
$$

By the methods in Solomyak's paper [7], it follows [6] that this power series has a transversality property, for $\lambda \in(0.5,0.6491-\varepsilon)$. One can use this to estimate that

$$
\int_{\frac{1}{2}+\varepsilon+\delta}^{0.6491-\varepsilon-\delta} I_{\left\{(a, b):\left|\rho(a)_{1}-\rho(b)_{1}\right|<r\right\}} \mathrm{d} \lambda<\operatorname{Cr}\left(\frac{1}{2}+\varepsilon+\delta\right)^{-n},
$$

where $C$ is a constant. Hence

$$
\begin{array}{rl}
J_{a_{-n} \cdots a_{0}, b_{-n} \cdots b_{0}} \leq \int_{\left[a_{-n} \cdots a_{0}\right] \times\left[b_{-n} \cdots b_{0}\right]} \operatorname{Cr}\left(\frac{1}{2}+\delta-\varepsilon\right)^{-n} & \mathrm{~d}\left(\mu_{\Sigma} \times \mu_{\Sigma}\right) \\
& =\operatorname{Cr}^{-2 n}\left(\frac{1}{2}+\varepsilon+\delta\right)^{-n}
\end{array}
$$

This implies that

$$
\begin{aligned}
I & \leq \liminf _{r \rightarrow 0} \frac{1}{2 r} \sum_{n=0}^{\infty} \sum_{a_{-n} \cdots a_{0}} \sum_{\substack{\left.b_{-n} \cdots b_{0} \\
a_{k}, b_{k} \in A_{0} \text { or } a_{k}, b_{k} \in A_{1}, k>-n \\
a_{-n}, b_{-n}\right\} \not \subset A_{0} \text { and }\left\{a_{-n}, b_{-n}\right\} \not \subset A_{1}}} \operatorname{Cr} 4^{-2 n}\left(\frac{1}{2}+\varepsilon+\delta\right)^{-n} \\
& =\frac{C}{2} \sum_{n=0}^{\infty} 4^{n} 2^{n} C 4^{-2 n}\left(\frac{1}{2}+\delta-\varepsilon\right)^{-n}=\frac{C}{2} \sum_{n=0}^{\infty}\left(\frac{1}{2\left(\frac{1}{2}+\varepsilon+\delta\right)}\right)^{n}<\infty,
\end{aligned}
$$

which finishes the proof.

\section{Proof of Theorem 3}

We will use the same notation as in the proof of Theorem 2. We first note that the $L_{2}$ norm of the density of $\nu_{\lambda, \varepsilon, m}$ is not larger than twice that of the density of $\mu_{\lambda, \varepsilon, m}$. Indeed, if $\mu_{\lambda, \varepsilon, m}$ is absolutely continuous with respect to Lebesgue measure, then so is $\nu_{\lambda, \varepsilon, m}$. If $h_{\nu_{\lambda, \varepsilon, m}}(x)$ and $h_{\mu_{\lambda, \varepsilon, m}}(x, y, z)$ denotes the density of $\nu_{\lambda, \varepsilon, m}$ and $\mu_{\lambda, \varepsilon, m}$ respectively, then by Lyapunov's inequality

$$
\begin{aligned}
\left\|\nu_{\lambda, \varepsilon, m}\right\|_{2}^{2} & =\int_{-1}^{1} h_{\nu_{\lambda, \varepsilon, m}}(x)^{2} \mathrm{~d} x \\
& =32 \int_{-1}^{1}\left(\int_{-1}^{1} \int_{-1}^{1} h_{\mu_{\lambda, \varepsilon, m}}(x, y, z) \frac{\mathrm{d} y}{2} \frac{\mathrm{d} z}{2}\right)^{2} \frac{\mathrm{d} x}{2} \\
& \leq 32 \int_{-1}^{1} \int_{-1}^{1} \int_{-1}^{1} h_{\mu_{\lambda, \varepsilon, m}}(x, y, z)^{2} \frac{\mathrm{d} y}{2} \frac{\mathrm{d} z}{2} \frac{\mathrm{d} x}{2}=4\left\|\mu_{\lambda, \varepsilon, m}\right\|_{2}^{2} .
\end{aligned}
$$


This proves that if $\mu_{\lambda, \varepsilon, m}$ has density in $L_{2}$, then so has $\nu_{\lambda, \varepsilon, m}$, and

$$
\left\|\nu_{\lambda, \varepsilon, m}\right\|_{2} \leq 2\left\|\mu_{\lambda, \varepsilon, m}\right\|_{2} .
$$

We will therefore bound the $L_{2}$ norm of $\mu_{\lambda, \varepsilon, m}$. For this purpose will need the following transversality property.

Lemma 3.I. The cones $C_{p}=\left\{(a, b, c) \in T_{p} Q:\left|\frac{a}{c}\right|,\left|\frac{b}{c}\right|<\frac{2^{m+2} \varepsilon}{2^{m}-\lambda-\varepsilon}\right\}, p \in Q$ defines a family of unstable cones, that is $\mathrm{d}_{p}\left(f_{\lambda, \varepsilon, m}\right)\left(C_{p}\right) \subset C_{f_{\lambda, \varepsilon, m}(p)}$.

Moreover, if $m$ is sufficiently large then the following is true. If $\gamma_{1} \subset Q_{\gamma_{1}}$ and $\gamma_{2} \subset Q_{\gamma_{2}}$ are two line segments with tangents in $C_{p}$, such that, $\gamma_{1} \in A_{0}$ and $\gamma_{2} \in A_{1}$, then if $f_{\lambda, \varepsilon, m}\left(\gamma_{1}\right)$ and $f_{\lambda, \varepsilon, m}\left(\gamma_{2}\right)$ intersect, and if $\left(a_{1}, b_{1}, 1\right)$ and $\left(a_{2}, b_{2}, 1\right)$ are tangents to $f_{\lambda, \varepsilon, m}^{k}\left(\gamma_{1}\right)$ and $f_{\lambda, \varepsilon, m}^{k}\left(\gamma_{2}\right)$ respectively, it holds $\left|a_{1}-a_{2}\right|>C_{\lambda, \varepsilon, m} 2^{-(k-1) m}(\lambda-\varepsilon)^{k-1} \varepsilon$, where

$$
C_{\lambda, \varepsilon, m}=\frac{2}{\lambda+\varepsilon}-\frac{4(\lambda+\varepsilon)}{2^{m}-\lambda-\varepsilon}>0 .
$$

Proof. One easily checks that $C_{p}$ defines a family of unstable cones: The Jacobian of $f_{\lambda, \varepsilon, m}$ is

$$
\left[\begin{array}{ccc}
\lambda_{0} & 0 & 2^{m+1}(x \pm 1) \varepsilon \\
0 & 2 & 0 \\
0 & 0 & 2^{m}
\end{array}\right]
$$

If $(a, b, c) \in C_{p}$, then

$$
\mathrm{d}_{p}\left(f_{\lambda, \varepsilon, m}\right)(a, b, c)=\left[\begin{array}{c}
\lambda_{0} a+2^{m+1}(x \pm 1) \varepsilon c \\
2 b \\
2^{m} c
\end{array}\right] .
$$

The estimates

$$
\begin{aligned}
\frac{\left|\lambda_{0} a+2^{m+1}(x \pm 1) \varepsilon c\right|}{\left|2^{m} c\right|} & \leq \frac{\lambda_{0}}{2^{m}} \frac{|a|}{|c|}+4 \varepsilon \leq \frac{\lambda+\varepsilon}{2^{m}} \frac{2^{m+2} \varepsilon}{2^{m}-\lambda-\varepsilon}+4 \varepsilon \\
& =\frac{2^{m+2} \varepsilon}{2^{m}-\lambda-\varepsilon}, \\
\frac{|2 b|}{\left|2^{m} c\right|} & <\frac{2^{m+2} \varepsilon}{2^{m}-\lambda-\varepsilon},
\end{aligned}
$$

proves that $\mathrm{d}_{p}\left(f_{\lambda, \varepsilon, m}\right)(a, b, c) \subset C_{f_{\lambda, \varepsilon, m}(p)}$.

Let us now prove the later part of the lemma. Recall that

$$
Q_{0}=\bigcup_{k=0}^{2^{m}-1} Q_{0, k}, \quad Q_{1}=\bigcup_{k=0}^{2^{m}-1} Q_{1, k}
$$

Take $p=(x, y, z) \in Q$. Then for $(a, b, 1) \in C_{p}$.

$$
\begin{aligned}
& p \in Q_{0} \quad \Rightarrow \quad \mathrm{d}_{p} f_{\lambda, \varepsilon, m}:(a, b, 1) \mapsto 2^{m}\left(\frac{\lambda_{0}}{2^{m}} a+(x+1) \varepsilon, \frac{b}{2^{m-1}}, 1\right), \\
& p \in Q_{1} \quad \Rightarrow \quad \mathrm{d}_{p} f_{\lambda, \varepsilon, m}:(a, b, 1) \mapsto 2^{m}\left(\frac{\lambda_{0}}{2^{m}} a+(x-1) \varepsilon, \frac{b}{2^{m-1}}, 1\right) .
\end{aligned}
$$


Assume that $p=\left(x_{p}, y_{p}, z_{p}\right) \in Q_{0}$, and $q=\left(x_{q}, y_{q}, z_{q}\right) \in Q_{1}$, are such that $f_{\lambda, \varepsilon, m}(p)=$ $f_{\lambda, \varepsilon, m}(q)=(x, y, z)$.

Then $x_{p}=\frac{x+\left(1-\lambda_{0}\right)}{\lambda_{0}}, x_{q}=\frac{x-\left(1-\lambda_{0}\right)}{\lambda_{0}}$ and

$$
\begin{aligned}
& \mathrm{d}_{p}\left(f_{\lambda, \varepsilon, m}\right)\left(C_{p}\right) \\
& \quad \subset\left\{c(a, b, 1): \frac{x+1}{\lambda+\varepsilon} \varepsilon-\frac{\lambda+\varepsilon}{2^{m}} \frac{2^{m+2} \varepsilon}{2^{m}-\lambda-\varepsilon}<a<\frac{x+1}{\lambda-\varepsilon} \varepsilon+\frac{\lambda+\varepsilon}{2^{m}} \frac{2^{m+2} \varepsilon}{2^{m}-\lambda-\varepsilon}\right\}, \\
& \mathrm{d}_{q}\left(f_{\lambda, \varepsilon, m}\right)\left(C_{q}\right) \\
& \quad \subset\left\{c(a, b, 1): \frac{x-1}{\lambda-\varepsilon} \varepsilon-\frac{\lambda+\varepsilon}{2^{m}} \frac{2^{m+2} \varepsilon}{2^{m}-\lambda-\varepsilon}<a<\frac{x-1}{\lambda+\varepsilon} \varepsilon+\frac{\lambda+\varepsilon}{2^{m}} \frac{2^{m+2} \varepsilon}{2^{m}-\lambda-\varepsilon}\right\} .
\end{aligned}
$$

Hence if $\left(a_{p}, b_{p}, 1\right) \in \mathrm{d}_{p}\left(f_{\lambda, \varepsilon, m}\right)\left(C_{p}\right)$ and $\left(a_{q}, b_{q}, 1\right) \in \mathrm{d}_{q}\left(f_{\lambda, \varepsilon, m}\right)\left(C_{q}\right)$ then

$$
\left|a_{p}-a_{q}\right|>\frac{2}{\lambda+\varepsilon} \varepsilon-\frac{8 \varepsilon(\lambda+\varepsilon)}{2^{m}-\lambda-\varepsilon}=C_{\lambda, \varepsilon, m} \varepsilon
$$

where

$$
C_{\lambda, \varepsilon, m}=\frac{2}{\lambda+\varepsilon}-\frac{8(\lambda+\varepsilon)}{2^{m}-\lambda-\varepsilon} .
$$

The constant $C_{\lambda, \varepsilon, m}$ is positive for sufficiently small $\varepsilon$ provided that

$$
\frac{2}{\lambda}-\frac{8 \lambda}{2^{m}-\lambda}>0 \quad \Leftrightarrow \quad 2^{m}>4 \lambda^{2}+\lambda,
$$

which is satisfied if $m$ is sufficiently large. This proves the lemma in the case $k=1$. The statement for $k>1$ follows by iteration of $\mathrm{d}\left(f_{\lambda, \varepsilon, m}\right)$.

The rest of the proof follows Tsujii's article [8]. For any $r>0$ we define the bilinear form $(\cdot, \cdot)_{r}$ of signed measures on $\mathbb{R}$ by

$$
\left(\rho_{1}, \rho_{2}\right)_{r}=\int_{\mathbb{R}} \rho_{1}\left(B_{r}(x)\right) \rho_{2}\left(B_{r}(x)\right) \mathrm{d} x,
$$

where $B_{r}(x)=[x-r, x+r]$. One easily proves, see [?], that if

$$
\liminf _{r \rightarrow \infty} \frac{1}{r^{2}}(\rho, \rho)_{r}<\infty,
$$

then the measure $\rho$ has density in $L_{2}(\mathbb{R})$ and

$$
\|\rho\|_{2}^{2} \leq \liminf _{r \rightarrow \infty} \frac{1}{r^{2}}(\rho, \rho)_{r},
$$

where $\|\rho\|_{2}$ denotes the $L_{2}$ norm of the density of $\rho$.

Let $\mu_{z}$ denote the conditional measure of $\mu_{\lambda, \varepsilon, m}$, conditioned on the set $R_{z}:=$ $\left\{(a, b, c) \in Q: b=b_{0}, c=z\right\}$, where $b_{0}$ is some number such that $-1<b_{0}<1$. Note that $\mu_{z}$ is independent of $b_{0}$ almost everywhere, so we can forget about $b_{0}$. 
Consider the quantity $J(r)=\frac{1}{r^{2}} \int_{-1}^{1}\left(\mu_{z}, \mu_{z}\right)_{r} \mathrm{~d} z$. We observe that the measure $\eta$ on $[-1,1]$, defined by $\eta(E)=\mu_{\lambda, \varepsilon, m}([-1,1] \times[-1,1] \times E)$ for any Borel set $E$, is proportional to Lebesgue measure on $[-1,1]$. This implies that

$$
\left\|\mu_{\lambda, \varepsilon, m}\right\|_{2}^{2}=\int_{-1}^{1}\left\|\mu_{z}\right\|_{2}^{2} \mathrm{~d} z \leq \liminf _{r \rightarrow 0} J(r)
$$

We will therefore estimate $J(r)$.

By the invariance of $\mu_{\lambda, \varepsilon, m}$ it follows that

$$
\mu_{z}=2^{-(m+1)} \sum_{a \in A} \mu_{f^{-a}(z)} \circ f^{-a},
$$

where $f^{-a}$ denotes the inverse of the function $f_{\lambda, \varepsilon, m}$ restricted to the cylinder $[a]$. By $f^{-a}(z)$, we mean the unique number $z_{a}$ such that there exists numbers $x_{a}, y_{a}, x, y$ with $\left(x_{a}, y_{a}, z_{a}\right) \in[a]$ and $f_{\lambda, \varepsilon, m}\left(x_{a}, y_{a}, z_{a}\right)=(x, y, z)$.

The formula (4) allow us to rewrite $J(r)$ as

$$
J(r)=\frac{1}{r^{2}} 2^{-2(m+1)} \sum_{a, b \in A} \int_{-1}^{1}\left(\mu_{f^{-a}(z)} \circ f^{-a}, \mu_{f^{-b}(z)} \circ f^{-b}\right)_{r} \mathrm{~d} z .
$$

For fixed $a$ and $b$ it holds

$$
\begin{aligned}
&\left(\mu_{f^{-a}(z)} \circ f^{-a}, \mu_{f^{-b}(z)} \circ f^{-b}\right)_{r} \\
& \leq\left(\mu_{f^{-a}(z)} \circ f^{-a}, \mu_{f^{-a}(z)} \circ f^{-a}\right)_{r}^{\frac{1}{2}}\left(\mu_{f^{-b}(z)} \circ f^{-b}, \mu_{f^{-b}(z)} \circ f^{-b}\right)_{r}^{\frac{1}{2}} \\
& \leq\left((\lambda+\varepsilon)\left(\mu_{f^{-a}(z)}, \mu_{f^{-a}(z)}\right)_{\frac{r}{\lambda-\varepsilon}}\right)^{\frac{1}{2}}\left((\lambda+\varepsilon)\left(\mu_{f^{-b}(z)}, \mu_{f^{-b}(z)}\right) \frac{r}{\lambda-\varepsilon}\right)^{\frac{1}{2}} \\
& \leq(\lambda+\varepsilon) \frac{\left(\mu_{f^{-a}(z)}, \mu_{f^{-a}(z)}\right) \frac{r}{\lambda-\varepsilon}+\left(\mu_{f^{-b}(z)}, \mu_{f^{-b}(z)}\right) \frac{r}{\lambda-\varepsilon}}{2} .
\end{aligned}
$$

Moreover

$$
\begin{aligned}
&\left(\mu_{f^{-a}(z)}\right.\left.\circ f^{-a}, \mu_{f^{-b}(z)} \circ f^{-b}\right)_{r} \\
&=\int \mu_{f^{-a}(z)} \circ f^{-a}\left(B_{r}(x)\right) \mu_{f^{-b}(z)} \circ f^{-b}\left(B_{r}(x)\right) \mathrm{d} x \\
&=\iiint I_{\{s:|s-x|<r\}}(s) I_{\{t:|t-x|<r\}}(t) \\
& \mathrm{d} \mu_{f^{-a}(z)} \circ f^{-a}(s) \mathrm{d} \mu_{f^{-b}(z)} \circ f^{-b}(t) \mathrm{d} x \\
& \leq \iint 2 r I_{\{(s, t):|s-t|<2 r\}}(s, t) \mathrm{d} \mu_{f^{-a}(z)} \circ f^{-a}(s) \mathrm{d} \mu_{f^{-b}(z)} \circ f^{-b}(t) \\
&=\iint 2 r I_{\left\{(c, d):\left|\rho^{-1}\left(\cdots c_{-2} c_{-1} a \rho_{0}(z)\right)-\rho^{-1}\left(\cdots d_{-2} d_{-1} b \rho_{0}(z)\right)\right|<2 r\right\}}(\boldsymbol{c}, \boldsymbol{d}) \\
& \quad \mathrm{d} \mu_{\Sigma}(\boldsymbol{c}) \mathrm{d} \mu_{\Sigma}(\boldsymbol{d}) .
\end{aligned}
$$


If $a$ and $b$ are such that there exists a $k$ such that either $a \in A_{0}, b \in A_{1}$ or $a \in A_{1}$, $b \in A_{0}$, then by Lemma 3.I and (7) we get that

$$
\begin{aligned}
& \int_{-1}^{1}\left(\mu_{f^{-a}(z)} \circ f^{-a}, \mu_{f^{-b}(z)} \circ f^{-b}\right)_{r} \mathrm{~d} z \\
& \quad \leq \iint 2 r \nu\left\{z:\left|\rho^{-1}\left(\cdots c_{-2} c_{-1} a \rho_{0}(z)\right)-\rho^{-1}\left(\cdots d_{-2} d_{-1} b \rho_{0}(z)\right)\right|<2 r\right\} \\
& \quad \leq 8 r^{2} \frac{1}{C_{\lambda, \varepsilon, m} \varepsilon},
\end{aligned}
$$

and so since there are at most $2^{2(m+1)}$ pairs $a, b$ such that either $a \in A_{0}, b \in A_{1}$ or $a \in A_{1}, b \in A_{0}$, we get that

$$
\sum_{\substack{a \in A_{0}, b \in A_{1} \\ \text { or } \\ a \in A_{1}, b \in A_{0}}} \int_{-1}^{1}\left(\mu_{f^{-a}(z)} \circ f^{-a}, \mu_{f^{-b}(z)} \circ f^{-b}\right)_{r} \mathrm{~d} z \leq \frac{8 r^{2}}{C_{\lambda, \varepsilon, m}} 2^{2(m+1)} .
$$

For the case that $a, b \in A_{0}$ or $a, b \in A_{1}$ we us (6) to estimate that

$$
\sum_{\substack{a, b \in A_{0} \\ a, b \in A_{1}}}\left(\mu_{f^{-a}(z)} \circ f^{-a}, \mu_{f^{-b}(z)} \circ f^{-b}\right)_{r} \leq(\lambda+\varepsilon) 2^{m} \sum_{a}\left(\mu_{f^{-a}(z)}, \mu_{f^{-a}(z)}\right) \frac{r}{\lambda-\varepsilon} .
$$

Now, (5) and the last two estimates implies

$$
\begin{aligned}
J(r) & \leq \frac{8}{C_{\lambda, \varepsilon, m} \varepsilon}+2^{-2(m+1)}(\lambda+\varepsilon) 2^{m} \frac{1}{r^{2}} \sum_{a} \int_{-1}^{1}\left(\mu_{f^{-a}(z)}, \mu_{f-a(z)}\right) \frac{r}{\lambda-\varepsilon} \mathrm{d} z \\
& =\frac{8}{C_{\lambda, \varepsilon, m} \varepsilon}+2^{-2(m+1)} \frac{\lambda+\varepsilon}{(\lambda-\varepsilon)^{2}} 2^{m} \frac{1}{\left(\frac{r}{\lambda-\varepsilon}\right)^{2}} \sum_{a} 2^{m} \int_{a}\left(\mu_{z}, \mu_{z}\right) \frac{r}{\lambda-\varepsilon} \mathrm{d} z \\
& =\frac{8 r^{2}}{C_{\lambda, \varepsilon, m} \varepsilon}+2^{-2}(\lambda-\varepsilon)^{-2}\left(\frac{\lambda+\varepsilon}{\lambda-\varepsilon}\right) J\left(\frac{r}{\lambda-\varepsilon}\right) .
\end{aligned}
$$

Hence

$$
\begin{aligned}
\frac{J(r)}{r^{2}} & \leq \frac{8}{C_{\lambda, \varepsilon, m} \varepsilon} \sum_{n=0}^{\infty}(2(\lambda-\varepsilon))^{-2 n}\left(\frac{\lambda+\varepsilon}{\lambda-\varepsilon}\right)^{n} \\
& =\frac{8}{C_{\lambda, \varepsilon, m} \varepsilon} \frac{(\lambda-\varepsilon)^{2}}{(\lambda-\varepsilon)^{2}-\frac{1}{4} \frac{\lambda+\varepsilon}{\lambda-\varepsilon}} .
\end{aligned}
$$

By (3) we thus have

$$
\left\|\mu_{\lambda, \varepsilon, m}\right\|_{2} \leq \frac{c_{m}}{\sqrt{\varepsilon}}
$$


with

$$
c_{m}=\sqrt{8 \frac{(\lambda-\varepsilon)^{2}}{(\lambda-\varepsilon)^{2}-\frac{1}{4} \frac{\lambda+\varepsilon}{\lambda-\varepsilon}}\left(\frac{2}{\lambda+\varepsilon}-\frac{4(\lambda+\varepsilon)}{2^{m}-\lambda-\varepsilon}\right)^{-1}} .
$$

By (1) we have

$$
\left\|\nu_{\lambda, \varepsilon, m}\right\|_{2} \leq 2\left\|\mu_{\lambda, \varepsilon, m}\right\|_{2} \leq \frac{c_{m}}{\sqrt{\varepsilon}} .
$$

Letting $m \rightarrow \infty$ we find that

$$
\left\|\nu_{\lambda, \varepsilon}\right\|_{2} \leq \frac{c}{\sqrt{\varepsilon}}
$$

with

$$
c=4 \sqrt{\frac{\left(\lambda^{2}-\varepsilon^{2}\right)(\lambda-\varepsilon)}{(\lambda-\varepsilon)^{2}-\frac{1}{4} \frac{\lambda+\varepsilon}{\lambda-\varepsilon}}}<\frac{4 \lambda \sqrt{\lambda}}{\sqrt{\lambda^{2}-\frac{1}{4}-(2 \lambda+1) \varepsilon}} .
$$




\section{References}

[I] P. Erdös, On a family of symmetric Bernoulli convolutions, American Journal of Mathematics 6I (1939), 974-976.

[2] P. Erdös, On the smoothness properties of a family of Bernoulli convolutions, American Journal of Mathematics 62 (1940), I80-I86.

[3] A. M. Garsia, Arithmetic properties of Bernoulli convolutions, Transactions of the American Mathematical Society I02 (1962), 409-432.

[4] Y. Peres, B. Solomyak, Absolute continuity of Bernoulli convolutions, a simple proof, Mathematical Research Letters 3 (1996), 23I-236.

[5] Y. Peres, W. Schlag, B. Solomyak, Sixty years of Bernoulli convolutions, Fractal geometry and stochastics, II (Greifswald/Koserow, 1998), 39-65, Progress in Probability 46, Birkhäuser, Basel, 2000.

[6] T. Persson, Absolutely continuous invariant measures for some piecewise hyperbolic affine maps, Ergodic Theory and Dynamical Systems 28:I, 21I-228.

[7] B. Solomyak, On the random series $\sum \pm \lambda^{i}$ (an Erdös problem), Annals of Mathematics I42 (1995), 6II-625.

[8] M. Tsujii, Fat solenoidal attractors, Nonlinearity I4:5 (200I), I0II-I027.

[9] A. Wintner, On Convergent Poisson Convolutions, American Journal of Mathematics $57: 4$ (1935), 827-838.

Tomas Persson, Centre for Mathematical Sciences, Box II8, SE-22100 Lund, Sweden tomasp@maths.lth.se, http://www.maths.lth.se/ tomasp 

Preprints in Mathematical Sciences 2008:7

ISSN 1403-9338

LUTFMA-5097-2008

Mathematics

Centre for Mathematical Sciences

Lund University

Box 118, SE-221 00 Lund, Sweden

http://www.maths.Ith.se/ 